\title{
Preparation of Hierarchical Mesoporous MFI-Type Zeolite Using Cost-Effective and Sustainable Hard Template
}

\author{
Mostafa $\mathrm{N}^{1 *}$, Shaban $\mathrm{SA}^{1}$, Osama AM${ }^{1}$, El Kady FY1 and El-Torgoman \\ $\mathrm{AM}^{2}$ \\ ${ }^{1}$ Catalysis Department, Refining Division, Egyptian Petroleum Research Institute, Egypt \\ ${ }^{2}$ Chemistry Department, Faculty of Science, Menofia University, Egypt
}

*Corresponding author: Nermen Mostafa, Catalysis Department, Refining Division,

Egyptian Petroleum Research Institute, 11727 Nasr City, Cairo, Egypt, Tel: 01015927813; Email: nermen_2088@yahoo.com

\section{Research Article}

Volume 3 Issue 4

Received Date: August 20, 2019

Published Date: August 30, 2019

DOI: $10.23880 /$ ppej- 16000201

\section{Abstract}

The preparation of hierarchical mesoporous zeolites has lately become one of the principal focuses in the catalysis field. In this work, hierarchical mesoporous ZSM-5 zeolite (HM-ZSM-5) was successfully synthesized using soluble starch as a cheap mesoprogen. Techniques such as XRD, N2 adsorption, FT-IR and TGA have been conducted to study the structural characteristics of the synthesized mesoporous ZSM-5 zeolite. The XRD and FTIR results verified the formation of aluminosilicate with MFI framework topology. Nitrogen isotherm of the HM-ZSM-5 sample showed a broad hysteresis loop can be in the relative vapor pressure range of 0.4-1.0 bar corroborating the generation of hierarchical mesoporosity in the synthesized zeolite sample. Additionally, the BJH pore-size distributions of HM-ZSM-5 confirmed the presence of hierarchical mesoporsity. The prepared HM-ZSM-5 sample has high thermal stability comparable to that of the conventional microporous counterpart. In light of its excellent properties including hierarchical porosity, large surface area as well as high thermal stability, HM-ZSM-5 can be deemed as a potentially promising catalyst with efficient masstransfer, reduced diffusion limitation and improved resistance against coke deactivation.

Keywords: ZSM-5; Hierarchical zeolites; Hard template; Starch

\section{Introduction}

Over the past decades, zeolites have been extensively used as efficient heterogeneous acid catalysts for a wide variety of important processes in the oil refining and petrochemicals industries [1-8]. These processes include fluid catalytic cracking (FCC), hydrocracking, xylene isomerization, alkylation of aromatics, reforming, linear paraffin isomerization and methanol-to-hydrocarbon conversions. The outstanding performance of zeolites as heterogeneous catalysts mainly originates from their strong Brønsted acidity and excellent shape selectivity $[3,9]$. 


\section{Petroleum \& Petrochemical Engineering Journal}

In spite of the advantages offered by zeolite microporsity in terms of the extremely high surface area and the unique shape selective properties, the sole presence of micropores induces severe mass transport limitations [8,9]. It causes inefficient utilization of the internal voids of zeolite [10-18]. The access of the bulky reactant molecules to the catalytically active sites located within them is hampered, thus the conversion of bulky reactants takes place only over the active sites located on the outer surfaces of the zeolite $[8,9,19,20]$. Additionally, it may also cause fast deactivation of the catalyst by pore plugging by carbonaceous residue (coke) $[8,20]$.

In recent years, two different strategies have been described in the literature to improve mass transfer in zeolite pores and increase accessibility to the catalytic active sites (see Figure 2). The first strategy is to shorten the diffusion path length for reactants and products via decreasing the crystal size of zeolite, the so-called nanosized zeolite crystals or nano-zeolites $[3,18]$. Although, nano-zeolites have been found to have superior catalytic performance, particularly when dealing with bulky molecules, and higher resistance against deactivation by coke formation as compared to their conventional micrometre-sized counterparts [21-25], their high synthesis cost and the problems encountered in their efficient separation from reaction media severely restrict their practical utility on a large-scale $[3,18,26]$.

The second strategy involves the introduction of a secondary mesoporous system i.e. pores with diameters ranging from 2.0 and $50 \mathrm{~nm}$, inside the microporous zeolite crystals $[3,8,18,19]$. The term hierarchical zeolites is commonly used in the literature to describe this type of zeolites because they have two levels of porosities; a supplementary mesoporoisty in addition to the original zeolite microporsity and thus having a hierarchy of pore sizes $[3,8,27]$. Hierarchically structured zeolite has gained enormous and increasing interest during recent years because they combine the positive aspects found in both microporous zeolites (shape-selectivity, strong acidity, high hydrothermal stability) and mesoporous materials (efficient mass-transfer, reduced diffusion limitation and improved resistance against coke deactivation) in one material. These properties make these materials suitable for catalytic applications which are beyond the reach of the conventional microporous zeolites or mesoporous materials $[3,8,18,27]$. Throughout the last several years, mesostrucured zeolites have proven to be highly efficient catalysts in a large variety of reactions, including selective hydrogenation [28], alkylation of benzene [29], conversion of methanol to hydrocarbon [30], xylene isomerization [31,32], hydrocracking [33] and fluid catalytic cracking [34].

Over the past few years, several methods for the preparation of hierarchical meso-structured zeolites have been reported. Generally, these methods can be categorized into three classes namely, assembly, demetallization and mixed methods. In the assembly methods, also called 'bottom-up' or 'constructive' methods, the mesoporous system is generated by mean of the self-assembly of nano-sized zeolite crystals in the presence of a sacrificial mesopore directing agent [8]. In the demetallization methods, also called 'top-down' or 'destructive' methods, mesoporosity is generated into preexisting microporous zeolites through the selective extraction of framework $\mathrm{Si}$ or $\mathrm{Al}$ atoms [8] and [35]. The third approach for the formation of hierarchically structured zeolites is the mixed methods or the zeolite recrystallization approach which is a combination of the above mentioned two methods. This route involves two steps: partial dissolution of a pre-synthesized microporous zeolite in an alkaline solution followed by the reassembling of the zeolitic fragments into a mesoporous structure in the presence of a surfactant [8] and [36].

In this work, hierarchical mesoporous ZSM-5 zeolite (HM-ZSM-5) was synthesized through the hard-template using strach as the mesoporous template. The structural characteristics of the HM-ZSM-5 were investigated by Xray powder diffraction (XRD), N2 sorption isotherm, Fourier Transform-Infrared Spectroscopy (FTIR) and thermal gravimetric analysis.

\section{Experimental}

\section{Preparation of Hierarchical Mesoporous ZSM-5}

A hierarchical mesoporous ZSM-5 (HM-ZSM-5) was synthesized using the tradition hydrothermal method [37]. Starch was used as a cheap mesopore template for modifying the pores of the catalyst. A typical synthesis was carried out as follows. In a $50 \mathrm{ml}$ propylene bottle; $0.083 \mathrm{~g}$ of $\mathrm{NaAlO}_{2}(\mathrm{Si} / \mathrm{Al}=20)$ was dissolved in $4.8 \mathrm{~g}$ TPAOH solution under vigorous stirring for $1 \mathrm{~h}$ to obtain a clear aluminate solution. $10.8 \mathrm{~g}$ deionized water and $0.032 \mathrm{~g} \mathrm{NaOH}$ were added to solution under stirring then $4.2 \mathrm{~g}$ TEOS was added dropwise into the resulting solution. The mixture was stirred for 4 hours at $353^{\circ} \mathrm{K}$ in an oil bath to obtain a uniform sol-gel, and then $3.5 \mathrm{~g}$ starch was added and stirred over night at $353^{\circ} \mathrm{K}$. Finally, the mixture was crystallized in a Teflon lined stainless steel autoclave at $453{ }^{\circ} \mathrm{K}$ for $48 \mathrm{~h}$. The solid product was 


\section{Petroleum \& Petrochemical Engineering Journal}

separated by centrifugation, washed to neutral with deionized water several times, dried overnight at $373^{\circ} \mathrm{K}$ and finally calcined in air at $873^{\circ} \mathrm{K}$ for $10 \mathrm{~h}$.

The H-form ZSM-5 sample was obtained as follows. Calcined ZSM-5 sample was put in $1 \mathrm{~mol} / \mathrm{L} \mathrm{NH} 4 \mathrm{OH}$ aqueous solution (with solid to liquid ratio of 1:40) and stirred for $6 \mathrm{~h}$ at $353^{\circ} \mathrm{K}$. Then, the $\mathrm{NH}_{4}$-type ZSM- 5 was separated and washed by deionized after three times of ion-exchange. Finally, the solid was dried overnight at $373^{\circ} \mathrm{K}$ and calcined in air at $873^{\circ} \mathrm{K}$ for $3 \mathrm{~h}$.

\section{Catalyst Characterization}

Powder X-ray diffraction (PXRD) patterns were recorded on a Bruker AXS D8-ADVANCE diffractometer using a filtered $\mathrm{CuK \alpha}$ radiation source $(\lambda=1.54056 \AA)$ operated at $40 \mathrm{kV}$ and $30 \mathrm{~mA}$. Diffraction data were collected in the $2 \theta$ range of $3-40^{\circ}$ with a $0.02^{\circ} 2 \theta$ step size and $0.4 \mathrm{~s}$ count time. The surface characteristics of the prepared samples were determined from $\mathrm{N}_{2}$ adsorptiondesorption isotherms measured at liquid nitrogen temperature $\left(-196^{\circ} \mathrm{C}\right)$ using a Quantachrome Nova $3200 \mathrm{~S}$ instrument. Before the measurements, the samples were degassed at $573^{\circ} \mathrm{K}$ under vacuum overnight to remove any guest molecule from catalyst surface and pores. The specific surface area was evaluated using the BET method applied to the range of relative pressures between 0.05 and 0.15 . The total pore volume was calculated using single-point adsorption method at a relative pressure of $\mathrm{P} / \mathrm{P}^{0}=0.95$. Fourier transform infrared (FT-IR) spectra were obtained on an ATI unicam (Mattson 936) Bench Top spectrometer with pressed $\mathrm{KBr}$ pellets in the range of $4000-400 \mathrm{~cm}^{-1}$.

\section{Results and discussion}

\section{XRD}

In the current investigation, hierarchical mesoporous ZSM-5 zeolites were synthesized using the co-templates of TPAOH and starch by adding starch in the conventional ZSM-5 synthetic gel. X-ray diffraction (XRD) diffractograms of the as-obtained HM-ZSM-5 sample is shown in Figure 1. The pattern of the micro-ZSM-5 sample prepared without the addition of starch is also given in the same figure for the sake of comparison. Both samples displayed diffraction lines at $2 \theta$ of $7.86^{\circ}, 8.78^{\circ}, 14.78^{\circ}$, $23.18^{\circ}, 23.90^{\circ}$ and $24.40^{\circ}$ which can be readily attributed to ZSM-5 zeolite (JCPDS no. 43-0321), suggesting that the two samples have the MFI framework topology.
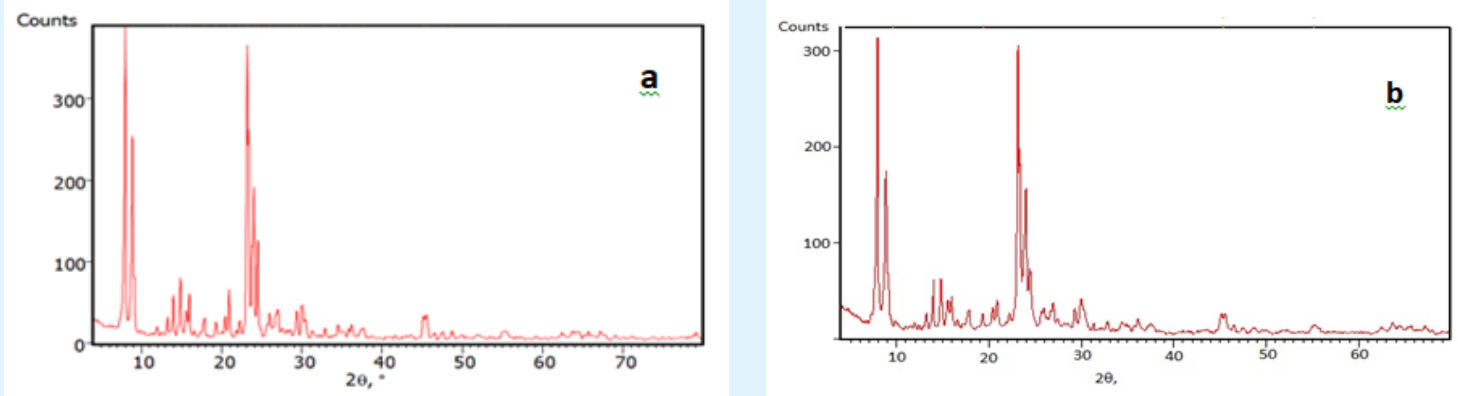

Figure 1: XRD patterns of micro-ZSM-5 (a) and hierarchical ZSM-5 (b).

\section{FTIR}

FT-IR spectra of the hierarchical and microporous ZSM- zeolite samples are depicted in Figure 2. The FTIR spectrum displayed a band at $3445 \mathrm{~cm}^{-1}$ that can be assigned to stretching siloxane groups or $\mathrm{O}-\mathrm{H}$ stretching of adsorbed water molecules [38,39]. The band at 1644 $\mathrm{cm}^{-1}$ can be indexed to physically adsorbed water [38]. The symmetric stretching of the siloxane groups appeared at $792 \mathrm{~cm}^{-1}$ [40]. The bands located at 545 and $447 \mathrm{~cm} 1$ are due to double five ring asymmetric stretching vibration and $\mathrm{Si}-\mathrm{O}-\mathrm{Si}$ bending mode [38,39], respectively. The two peaks at 1225 and $1102 \mathrm{~cm}^{-1}$ stand for the external and internal asymmetric stretching vibration of the siloxane groups $[38,40]$. The FTIR spectra of the samples verified the formation of aluminosilicate MFI structure, in line with XRD results.

\section{SSA}

In order to fully understand the porous features of the synthesized zeolites, nitrogen sorption analysis at $-196^{\circ} \mathrm{C}$ was conducted and the obtained isotherms are illustrated in Figure 3 together with the Barrett-Joyner-Halenda. (BJH) pore size distribution plots. As is shown in Fig. 3a, micro-ZSM-5 sample presented a typical type I isotherm 


\section{Petroleum \& Petrochemical Engineering Journal}

characteristic of microporous materials according to IUPAC classification of adsorption isotherms. This was further confirmed by the $\mathrm{BJH}$ pore size distribution plot (Fig. 3b), where no peak can be observed in the mesoporous range, indicating the sole presence of microporosity in the micro-ZSM-5 sample prepared without the addition of starch in the synthesis gel. The micro-ZSM-5 has BET specific
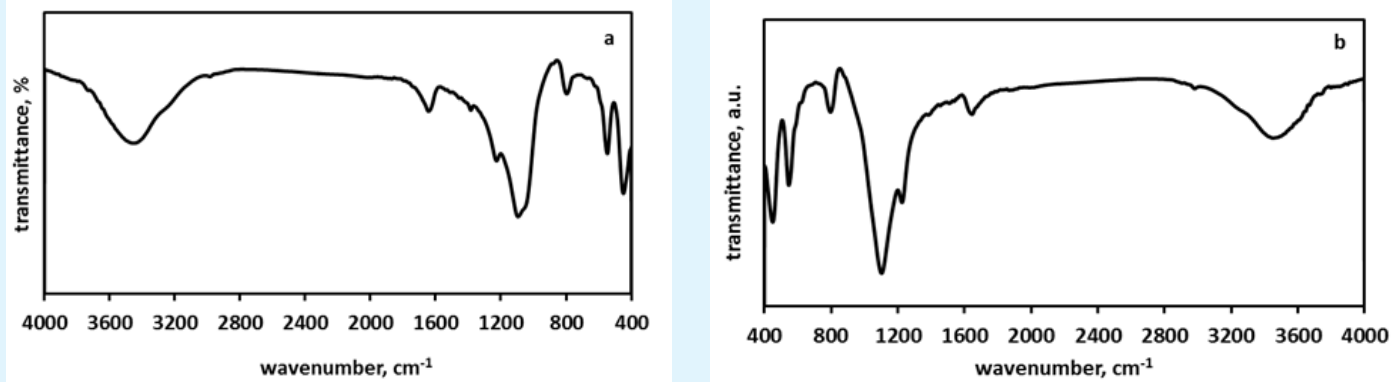

Figure 2: FTIR spectra of micro-ZSM-5 (a) and hierarchical ZSM- (b).

Surface area and total pore volume of about $311 \mathrm{~m}^{2} \mathrm{~g}-1$ and $0.15 \mathrm{~cm}^{3} \mathrm{~g}^{-1}$, respectively, with low mesoporous surface area and pore volume of $31 \mathrm{~m}^{2} \mathrm{~g}^{-1}$ and $0.015 \mathrm{~cm}^{3} \mathrm{~g}$ 1 , respectively. On the other hand, the obtained adsorption isotherm of the hierarchical ZSM-5 sample (Fig. 3c) can be deemed as an intermediate between Type-I and Type-IV isotherms, based on the IUPAC classification of sorption isotherms, indicating the coexistence of both micro- and mesoporosity. The amount of adsorbed nitrogen was found to increase dramatically in the low relative vapor pressure region of the isotherm demonstrating the existence of microporosity. Additionally, broad hysteresis loop can be also seen in the relative vapor pressure range of 0.4-1.0 bar, thus corroborating the generation of hierarchical mesoporosity in zeolites by employing starch as a mesopore-forming template. Additionally, the $\mathrm{BJH}$ pore-size distributions of HM-ZSM-5 (Fig. 3d) confirmed the presence of hierarchical mesoporsity.
The hierarchical sample displayed much larger mesoporous surface area $\left(409 \mathrm{~m}^{2} / \mathrm{g}\right)$ and pore volume $\left(0.315 \mathrm{~cm}^{3} / \mathrm{g}\right)$ as compared to the conventional microZSM-5. The hierarchical sample displayed much larger mesoporous pore volumes $\left(0.173 \mathrm{~cm}^{3} / \mathrm{g}\right)$ as compared to the conventional micro-ZSM-5 $\left(0.015 \mathrm{~cm}^{3} / \mathrm{g}\right)$.

\section{The Thermal Gravimetric Analysis (TGA)}

The thermal gravimetric analysis (TGA) of hierarchical and microporous ZSM- zeolite samples under $\mathrm{N}_{2}$ gas flow was conducted to study the effect of introducing mesoporosity on the thermal stability of the obtained hierarchical zeolite and the results are shown in Figure 4. As can be seen in this figure both samples have almost the same thermal stabilities, in other words the introduction of secondary mesoporous system has no adverse effect on the thermal stability of zeolite.
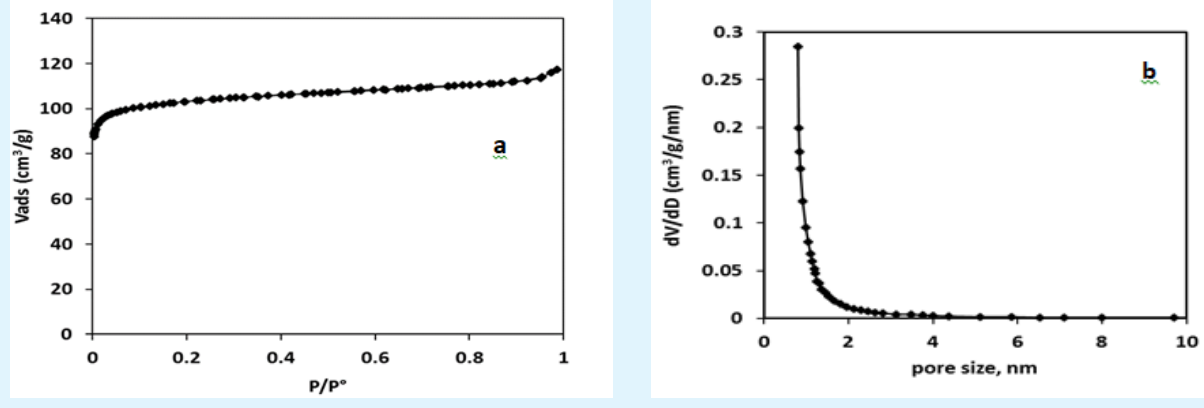


\section{Petroleum \& Petrochemical Engineering Journal}
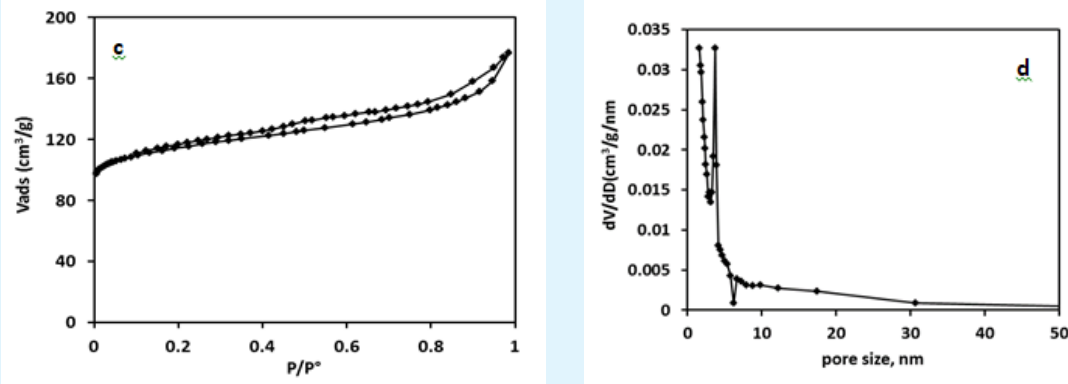

Figure 3: Nitrogen adsorption isotherms and BJH pore size distribution curves of micro-ZSM-5 $(\mathrm{a}, \mathrm{b})$ and hierarchical ZSM-5 (c,d).
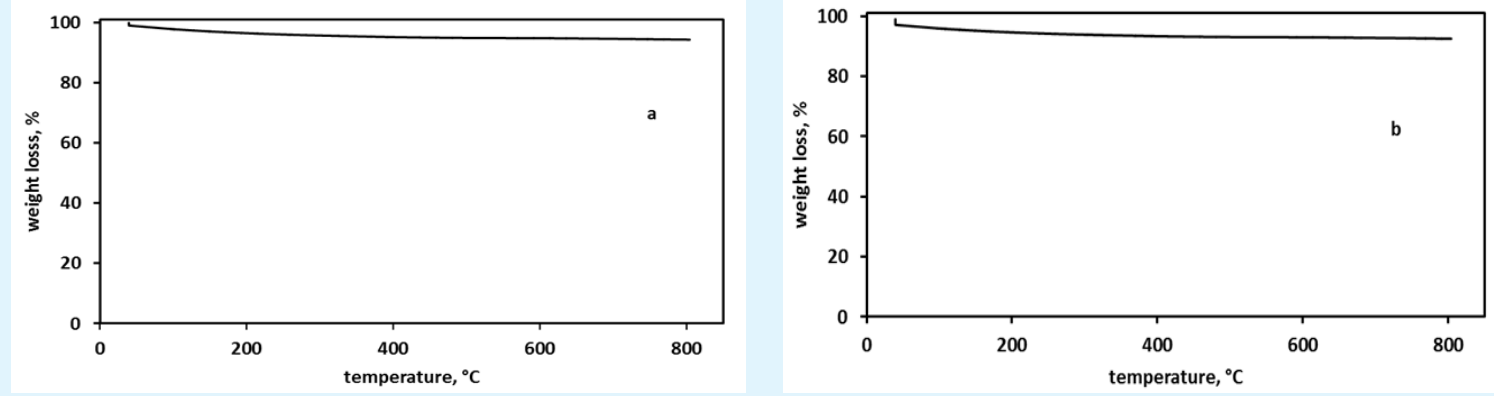

Figure 4: TGA curves of micro-ZSM-5 (a) and hierarchical ZSM-5 (b).

\section{Conclusions}

In the present work, we report the synthesis of hierarchical mesoporous ZSM-5 zeolite through the hardtemplate approach using soluble starch as a cheap, sustainable and available mesoprogen. The obtained hierarchical mesoporous ZSM-5 zeolite exhibited high surface area, hierarchical porosity, and excellent thermal stability. These properties make HM-ZSM-5 suitable for catalytic applications which are beyond the reach of the conventional microporous zeolites.

\section{References}

1. Wang Z, Yu J, Xu R (2012) Needs and trends in rational synthesis of zeolitic materials. Chem Soc Rev 41(5): 1729-1741.

2. Li K, Valla J, Garcia-Martinez J (2014) Realizing the Commercial Potential of Hierarchical Zeolites: New Opportunities in Catalytic Cracking. Chem Cat Chem 6(1): 46-66.
3. Na K, Choi M, Ryoo R (2013) Recent advances in the synthesis of hierarchically nanoporous zeolites Micropor Mesopor Mat 166: 3-19.

4. Youssef HF, Hegazy WH, Abo-almaged HH, ElBassyouni GT (2015) Novel Synthesis Method of Micronized Ti-Zeolite Na-A and Cytotoxic Activity of Its Silver Exchanged Form. Bioinorg Chem Appl 2015: 428121.

5. Fuoco D (2012) A New Method for Characterization of Natural Zeolites and Organic Nanostructure Using Atomic Force Microscopy. Nanomaterials 2(1): 79-91.

6. Yilmaz B, Trukhan N, Muller U (2012) Industrial Outlook on Zeolites and Metal Organic Frameworks. Chin J Catal 33(1): 3-10.

7. Colombo V, Galli S, Choi HJ, Han GD, Maspero A, et al. (2011) High thermal and chemical stability in pyrazolate-bridged metal-organic frameworks with exposed metal sites. Chem Sci 2: 1311-1319. 


\section{Petroleum \& Petrochemical Engineering Journal}

8. Wei Y, Parmentier TE, de Jong KP, Zecevic J (2015) Tailoring and visualizing the pore architecture of hierarchical zeolites. Chem Soc Rev 44: 7234-7261.

9. Srivastava R, Choi M, Ryoo R (2006) Mesoporous materials with zeolite framework: remarkable effect of the hierarchical structure for retardation of catalyst deactivation. Chem Comm 2006(43): 44894491.

10. Smit B, Maesen TL (2008) Towards a molecular understanding of shape selectivity. Nature 451(7179): 671-678.

11. Tavolaro A, Riccio II, Tavolaro P (2013) Hydrothermal synthesis of zeolite composite membranes and crystals as potential vectors for drugdelivering biomaterials. Micropor Mesopor Mater 167: 62-70.

12. Aguado J, Serrano DP (1999) Feedstock recycling of plastic wastes. RSC, UK, pp: 131.

13. Weisz PB, Frilette VJ (1960) Intracrystalline and Molecular-Shape-Selective Catalysis by Zeolite Salts. J Phys Chem 64(3): 382.

14. Wang Z, Yu J, Xu R (2012) Needs and trends in rational synthesis of zeolitic materials. Chem Soc Rev 41: 1729-1741.

15. Csicsery SM (1986) Catalysis by shape selective zeolites-science and technology. Pure \& App Chem 58(6): 841-856.

16. Beyer EMHK, Karge HG, Kiricsi I, Nagy JB (1995) Catalysis by Microporous Materials. 1st(Edn.), Vol. 94, Elsevier, Amsterdam.

17. Chen NY, Degnan TF, Smith CM (1994) Molecular Transport and Reaction in Zeolites: Design and Application of Shape Selective Catalysts. VCH publisher, New York, pp: 328.

18. Chen L, Li X, Rooke JC, Zhang Y, Yang X, et al. (2012) Hierarchically structured zeolites: synthesis, mass transport properties and applications. J Mater Chem 22: 17381-17403.

19. Meng X, Nawaz F, Xiao F (2009) Templating route for synthesizing mesoporous zeolites with improved catalytic properties. Nano Today 4(4): 292-301.
20. Moller K, Bein T (2013) Mesoporosity-a new dimension for zeolites. Chem Soc Rev 42(9): 36893707.

21. Smit B, Maesen TL (2008) Towards a molecular understanding of shape selectivity. Nature 451(7179): 671-678.

22. Kresge CT, Leonowicz ME, Roth WJ, Vartuli JC, Beck JS (1992) Ordered mesoporous molecular sieves synthesized by a liquid-crystal template mechanism. Nature 359: 710-712.

23. Chal R, Gerardin C, Bulut M, Van Donk S (2011) Overview and Industrial Assessment of Synthesis Strategies towards Zeolites with Mesopores. ChemCatChem 3(1): 67-81.

24. Xie R, Wang $\mathrm{H}$, Gao $\mathrm{P}$, Xia L, Zhang Z, et al. (2015) Core@shell $\mathrm{Co}_{3} \mathrm{O}_{4} @ \mathrm{C}-\mathrm{m}-\mathrm{SiO}_{2}$ catalysts with inert C modified mesoporous channel for desired middle distillate. Appl Catal A 492: 93-99.

25. Camblor MA, Corma A, Martinez A, Mocholi FA, Pariente JP (1989) Catalytic cracking of gasoil: Benefits in activity and selectivity of small Y zeolite crystallites stabilized by a higher silicon-toaluminium ratio by synthesis. Appl Catal 55(1): 6574.

26. Chen LH, Li XY, Tian G, Li Y, Rooke JC, et al. (2011) Highly Stable and Reusable Multimodal Zeolite TS-1 Based Catalysts with Hierarchically Interconnected Three-Level Micro-Meso-Macroporous Structure. Angew Chem Int Edit 50(47): 11156-11161.

27. Liu J, Jiang G, Liu Y, Di J, Wang Y, et al. (2014) Hierarchical Macro-meso-microporous ZSM-5 Zeolite Hollow Fibers With Highly Efficient Catalytic Cracking Capability. Sci Rep 4: 7276.

28. Hunns JA, Arroyo M, Lee AF, Serrano D, Wilson K, et al. (2016) Hierarchical mesoporous Pd/ZSM-5 for the selective catalytic hydrodeoxygenation of m-cresol to methylcyclohexane. Catal Sci Technol 6: 2560-2564.

29. Liu H, Liu S, Xie S, Song C, Xin W, et al. (2015) Effect of Desilication on the Performance of Hierarchical ZSM11 Catalysts for Alkylation of Benzene with Dimethyl Ether. Catal Lett 145(11): 1972-1983.

30. Liu Z, Dong X, Zhu Y, Emwas AH, Zhang D, et al. (2015) Investigating the Influence of Mesoporosity in Zeolite Beta on Its Catalytic Performance for the 


\section{Petroleum \& Petrochemical Engineering Journal}

Conversion of Methanol to Hydrocarbons. ACS Catal 5(10): 5837-5845.

31. Fernandez C, Stan I, Gilson JP, Thomas K, Vicente A, et al. (2010) Hierarchical ZSM-5 Zeolites in Shape-Selective Xylene Isomerization: Role of Mesoporosity and Acid Site Speciation. Chem A Euro J 16(21): 6224-6233.

32. Jian ZHOU, Zhicheng LIU, Liyuan LI, Yangdong WANG, Huanxin GAO, et al. (2013) Hierarchical mesoporous ZSM-5 zeolite with increased external surface acid sites and high catalytic performance in o-xylene isomerization. Chin J Catal 34(7): 1429-1433.

33. de Jong KP, Zečević J, Friedrich H, de Jongh PE, Bulut M, et al. (2010) Zeolite Y Crystals with Trimodal Porosity as Ideal Hydrocracking Catalysts. Angew Chem 122(52): 10272-1076.

34. Zhao J, Yin Y, Li Y, Chen W, Liu B (2016b) Synthesis and characterization of mesoporous zeolite $\mathrm{Y}$ by using block copolymers as templates. Chem Eng J 284: 405-411.
35. Moliner M (2012) Direct Synthesis of Functional Zeolitic Materials. Materials Science pp: 24.

36. Sachse A, Garcia Martinez J (2017) SurfactantTemplating of Zeolites: From Design to Application. Chem Mater 29(9): 3827-3853.

37. Tao H, Li C, Ren J, Wang Y, Lu G (2011) Synthesis of mesoporous zeolite single crystals with cheap porogens. Journal of Solid State Chemistry 184(7): 1820-1827.

38. Vinícius PS Caldeira, Anne GD Santos, Sibele BC Pergher, Maria JF Costa Antonio S (2016) Use of a low-cost template-free zsm-5 for atmospheric petroleum residue pyrolysis. New Chemistry 39(3).

39. Xin H, Koekkoek A, Yang Q, van Santen R, Li C, et al. (2009) Chemical Communications (48): 7590-7592.

40. Bleken BT1, Mino L, Giordanino F, Beato P, Svelle S, et al. (2013) Probing the surface of nanosheet H-ZSM-5 with FTIR spectroscopy. Phys Chem Chem Phys 15(32): 13363-13370. 